\title{
Determining the progenitors of supernovae with early robotic observations
}

\author{
Andrew Howell ${ }^{1,2}$ \\ ${ }^{1}$ Las Cumbres Observatory Global Telescope Network, 6740 Cortona Dr., Suite 102, Goleta, \\ CA, 93117, USA \\ ${ }^{2}$ Department of Physics, Broida Hall, University of California, Santa Barbara, CA 93106-9530 \\ email: ahowell@lcogt.net
}

\begin{abstract}
We present results from the LCOGT Supernova Key Project, a three year program to obtain lightcurves and spectra of 600 supernovae. The Las Cumbres Observatory Global Telescope Network is a network of eleven robotic $1 \mathrm{~m}$ and $2 \mathrm{~m}$ telescopes located at 5 sites around the world. With this facility long term monitoring of transient phenomena is possible, as are nearly instantaneous observations. We report on both core-collapse and thermonuclear supernovae observed within days of explosion, allowing insight into their progenitor stars.
\end{abstract}

